\title{
p53-Based cyclotherapy: exploiting the 'guardian of the genome' to protect normal cells from cytotoxic therapy
}

\author{
B Rao ${ }^{1}$, S Lain ${ }^{2}$ and A M Thompson*,1 \\ ${ }^{1}$ Dundee Cancer Centre, University of Dundee; Ninewells Hospital and Medical School, Dundee, UK and ${ }^{2}$ Microbiology, \\ Tumor and Cell Biology, Karolinska Institutet, Stockholm, Sweden
}

\begin{abstract}
Side effects of chemotherapy are a major impediment in the treatment of cancer. Cyclotherapy is an emerging therapeutic strategy for protecting normal cells from the side effects of chemotherapy. Low, non-genotoxic doses of known p53 activators can be used to induce p53-dependent cell cycle arrest in normal cells bearing wild-type p53. This cytostatic effect of p53 can protect normal cells from the toxicity of S- or M-phase poisons. Here, we have reviewed existing cyclotherapy regimens using two well-known p53 activators, nutlin-3 and actinomycin D. We have highlighted an exemplar clinical perspective for cyclotherapy in breast cancer. The recent development of novel stapled peptides as activators of p53 without the corresponding cytotoxicity holds great promise for cyclotherapy to enhance the therapeutic window of existing chemotherapy drugs.
\end{abstract}

Current treatments for cancer comprise surgery, radiotherapy, chemotherapy, endocrine therapy and targeted therapies. Modern chemotherapy is a systemic approach to treat cancer compared with the locoregional approaches of surgery and radiation therapy, and uses chemical agents (anticancer or cytotoxic drugs) to interact with cancer cells and eradicate or control the growth of cancer.

The purpose of the chemotherapy is to selectively kill tumour cells. Approximately 50 different anticancer drugs are available for clinical practice and an equal number are currently being tested in clinical trials. The most desirable drug will be one that destroys the cancer cells, leaves normal cells unharmed, and causes the fewest unpleasant and undesirable side effects. The therapeutic goal is to favourably balance the risk-benefit ratio, in which the morbidity and toxicity of chemotherapeutic agents are weighed against the potential benefits. Classic chemotherapeutic agents preferentially target rapidly dividing cells, whether cancer cells or normal cells. Therefore, selectivity towards cancer cells by standard chemotherapeutic drugs is limited and toxicity to normal tissues remains a therapeutically limiting problem in the clinic.

Suppression of bone marrow activity, which results in a decrease in white cell, platelet and red blood cell production, is a key limiting factor in standard chemotherapy. Because chemotherapy is most effective when used at the highest tolerated dose, the interval between treatments may need to be prolonged to prevent life-threatening bone marrow suppression. Supportive measures such as prophylaxis for or treatment of bone marrow suppression include granulocyctestimulating factors and blood product transfusion. Other undesired consequences of standard chemotherapy include hair loss, mucositis, gastrointestinal tract toxicity, dermatological toxicity, cardiotoxicity and asthenia. In addition, most classic chemotherapeutics are highly mutagenic either by causing damage to DNA (directly or indirectly) or, as in the case of mitotic poisons, by disrupting chromosome distribution. This can lead to an increased risk of second tumours later in life for those who survive the cancer.

\section{P53-BASED CYCLOTHERAPY}

Given the clinical problem of differential chemotherapeutic effects on tumour vs normal cells, cyclotherapy is a promising strategy to protect normal tissues from the side effects of chemotherapy (Blagosklonny and Pardee, 2001). Here, we briefly review key facets of the $p 53$ gene and focus on $p 53$-based cyclotherapy. This approach exploits the key genetic difference of the $p 53$ status between a normal cell and a p53 mutant bearing cancer cell and therefore is relevant to patients suffering from cancers carrying inactivating mutations in the $p 53$ gene.

*Correspondence: Professor AM Thompson; E-mail: a.m.thompson@dundee.ac.uk

Received 28 September 2012; revised 16 April 2012; accepted 1 May 2013;

published online 14 November 2013

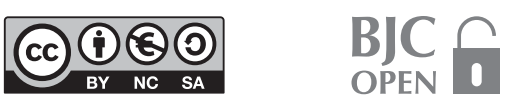


As shown in Figure 1, p53 activation by low doses of p53 smallmolecule activators can be used to selectively guard normal cells from the killing effects and genomic instability induced by two specific types of standard chemotherapeutics (S-phase and mitotic poisons). Small-molecule $p 53$ activators have no effect on p53 mutant bearing cancer cells, so these cells remain susceptible to the $\mathrm{S}$ phase or $\mathrm{M}$ phase targeting chemotherapeutic drug.

Activation of wild-type $p 53$ in normal tissues may cause p53-related toxicities. For p53-based cyclotherapy, only low doses of $p 53$ activators are used to specifically achieve the 'cytostatic' rather than the 'cytotoxic' effects of $p 53$ activation in normal tissues.

\section{THE REVERSIBLE CYTOSTATIC EFFECTS OF P53}

The p53 gene, also known as 'The Guardian of the Genome' (Lane, 1992), is located on the short arm of chromosome 17 (17p13.1) (Isobe et al, 1986). The inheritance of only one functional copy of the p53 gene causes a familial syndrome called Li-Fraumeni syndrome and these patients are predisposed to cancer (Malkin et al, 1990). Studies on p53-null mice show that although viable (and seemingly normal), they are prone to early tumour development (Donehower et al, 1996). A major biological function of $p 53$ is to respond to stress signals and activate the transcription of downstream target genes involved in important cellular mechanisms like cell cycle control, DNA repair and apoptosis. For the cell cycle control mechanisms, $p 53$ has two very distinct roles. The first is a protective (cytostatic) one in which p53 arrests cells in the G1 phase of the cell cycle upon sensing DNA damage. p53 thereby prevents cells from multiplying damaged DNA via the production of $\mathrm{p} 21$, which interacts with a cell division-stimulating protein $(\mathrm{cdk} 2)$. With $\mathrm{p} 21$ bound to $\mathrm{cdk} 2$, a cell cannot pass through to the next phase of the cell cycle (Figure 2). In the absence of functional $p 53, \mathrm{p} 21$ protein is not increased and cannot act as the 'stop signal' for cell division. In a second role, p53 initiates apoptosis upon irreparable damage to the cell.

Various different cellular signals like stress due to DNA damage, activation of oncogenes, hypoxia and nutrient deprivation can

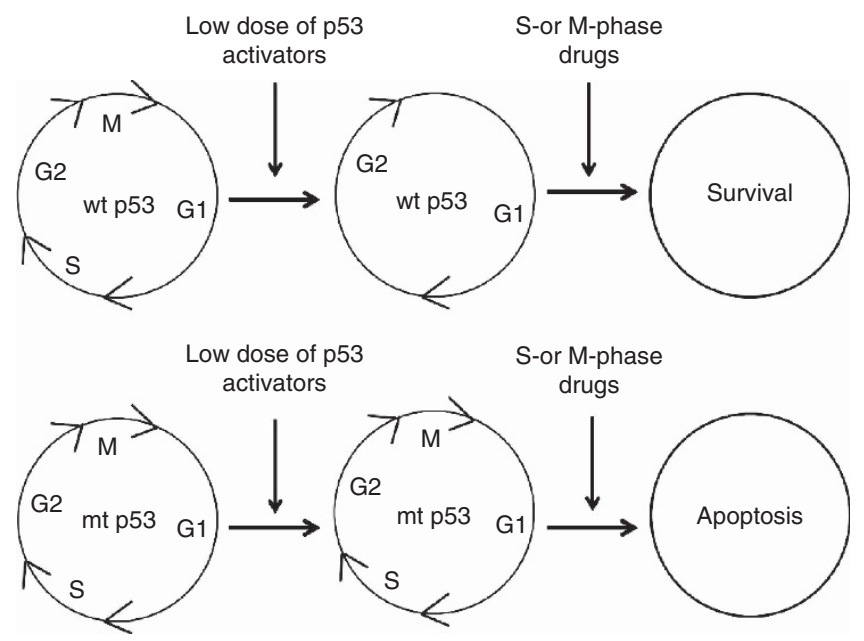

Figure 1. Principle of p53-based cyclotherapy: cyclotherapy, unlike conventional chemotherapy, distinguishes cancer cells from normal cells. Pretreatment with a p53 activating drug halts the cell cycle in G1/G2 only in normal cells carrying wild-type p53, whereas p53-mutant cancer cells continue cycling into $\mathrm{S}$ and $\mathrm{M}$ phase. Adding an S-phase or M-phase poison should then specifically target p53-mutant cancer cells to bring about apoptosis, while having no effect on cell cycle-arrested normal cells. induce p53 transcriptional activity. The specific response of p53 to these different cellular stresses depends on post translational modifications like phosphorylation and acetylation. In addition, it also depends on p53 interaction with its partners such as Mdm2. p53 levels are tightly regulated by $\mathrm{Mdm} 2$, an E3 ubiquitin ligase that causes proteasomal degradation of p53 (Toledo and Wahl, 2006). Interestingly, p53 protein transcriptionally activates Mdm2 to form a negative feedback mechanism, which maintains low p53 levels under normal, unstressed conditions. During stress activation, for example DNA damage, ATM/ATR kinases phosphorylate both $\mathrm{p} 53$ and $\mathrm{Mdm} 2$ proteins causing disruption in the interaction between the two. This phosphorylation facilitates p53 protein stabilisation leading to the transactivation of p53 target genes (reviewed in Toledo and Wahl, 2006). During oncogene activation, induction of another tumour suppressor protein p14ARF (known as p19ARF in mice) can also cause p53 protein stabilisation as p14ARF has been directly shown to bind Mdm2 and prevent the p53 degradation (Weber et al, 1999).

DNA damage has been shown to cause p53 transcriptional activity resulting in p21 induction that can result in both $\mathrm{G} 1$ and G2 arrest (Vogelstein et al, 2000). Even low levels of DNA damage can cause G2 arrest and non-entry into $M$ phase in p53 wild-type HCT116 cells (Bunz et al, 1998). Inducible systems have demonstrated that p53 overexpression can result in both G1 and G2 arrest, and that prolonged overexpression of p53 could downregulate Cyclin B1 (Agarwal et al, 1995). As the Cyclin $\mathrm{B} 1 / \mathrm{Cdc} 2$ complex is probably the major regulatory factor required for entry into mitosis, decreased expression of Cyclin B1 and inhibitory phosphorylation of Cdc2 are thought to be the major mediators of G2 arrest. Overexpression of p21 also causes cells to accumulate in G2 and is associated with a reduction of Cyclin B1-associated kinase activity (Niculescu et al, 1998). Expression of p21 may also be associated with a brief delay in G2, probably causing late cell cycle checkpoints in normal cycling cells (Dulic et al, 1998).

\section{P53-BASED CYCLOTHERAPY USING NUTLIN-3}

p53-deficient or p21-deficient HCT116 colon cancer cells enter mitosis despite pretreatment with doxorubicin, a DNA-damaging drug. This pretreatment allowed for the protection of only the wild-type $p 53$ HCT116 cells from the cell death induced by taxol (paclitaxel) and not the p53- or p21-deficient HCT116 cells (Blagosklonny et al, 2000). However, studies have also shown that pretreating with doxorubicin can also induce growth arrest in some p53-deficient tumours, hence protecting them from taxol (Blagosklonny, 2002). This undesired effect of doxorubicin suggested that, more $p 53$-selective compounds are required for cyclotherapy to be a successful approach.

Nutlin-3 has been the forefront candidate for p53-based cyclotherapy. Nutlins are a group of cis-imidazoline analogues, first identified by Vassilev et al (2004). These compounds have a high binding potency and selectivity for one of the p53 binding sites on Mdm2. Crystallisation data have shown that nutlin-3 mimics the three residues of the helical region of the transactivation domain of p53 (Phe19, Trp23 and Leu26) that are conserved across species and critical for binding to $\mathrm{Mdm} 2$. In this way, nutlin-3 prevents effective binding of p53 to Mdm2.

Two different groups demonstrated the protective role of nutlin3 via the p53/Mdm2 mechanisms using the isogenic colon cancer cell lines HCT116 and HCT116p53-/- (Carvajal et al, 2005; Kranz and Dobbelstein, 2006). The first was a sequential combination of nutlin-3 and taxol, a well-known and widely used mitotic inhibitor. Pretreating wild-type p53 cells with nutlin-3 before adding taxol caused these cells to arrest in G1 or G2 of the 
A

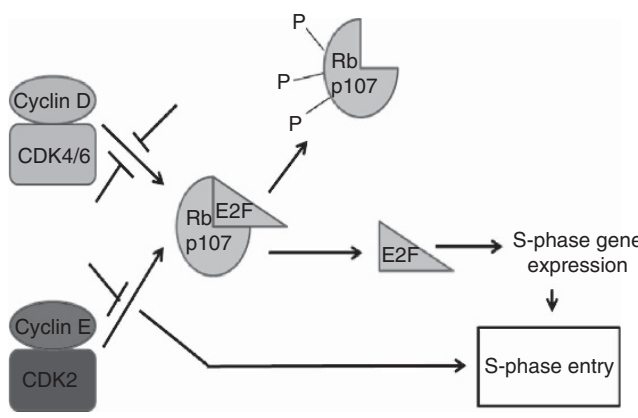

B

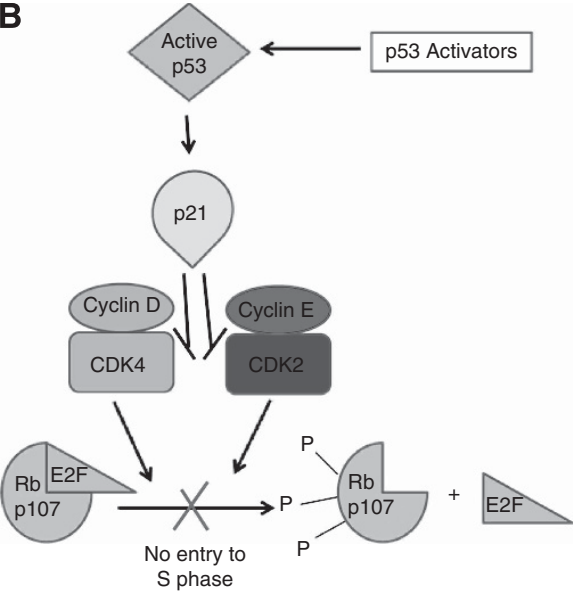

Figure 2. (A) Schematic to show the processes involved during S-phase entry. Rb phosphorylation is an important step during the regulation of normal cell entry to the S phase. (B) Upon addition of a low dose of p53 activating drug, as a result of p53 transcriptional activity, the cdk inhibitor p21 is activated. p21 transcription results in Rb hypophosphorylation via CDK4 and CDK2 inhibition leading to growth arrest. Cells would then not gain entry into $\mathrm{S}$ phase.

cell cycle, hence protecting them from taxol-induced apoptosis. This arrest was due to p53-dependent activation of p21 only upon pretreatment with nutlin-3 or its active enantiomer nutlin-3a and only when cells encoded wild-type $p 53$. In addition, sequential combination also protected proliferating skin fibroblasts from taxol with this arrest reversible upon drug removal (Carvajal et al, 2005).

Subsequently, a similar sequential combination was carried out and cells carrying functional $p 53$ were shown to be protected by nutlin-3 pretreatment from the cytotoxicity of the S-phase inhibitor gemcitabine. In contrast, isogenic cells lacking functional p53 continued to enter S-phase regardless of nutlin-3 pretreatment and remained highly susceptible to gemcitabine-mediated cytotoxicity. The sequential treatment with nutlin-3 alone, followed by transient exposure to nutlin-3 plus gemcitabine, efficiently killed tumour cells with deletions or mutations of $p 53$ but largely spared the non-transformed human keratinocytes (Kranz and Dobbelstein, 2006). The positive results described in these two cell culture studies (Carvajal et al, 2005; Kranz and Dobbelstein, 2006) have been confirmed and extended by experiments using two breast cancer cell lines expressing mutant p53 (van Leeuwen et al, 2012).

To date, there is only one report of a p53-based cyclotherapy study in vivo. In this study, nutlin-3 pretreatment was shown to protect mice from neutropaenia induced by the Polo-Like-Kinase-1 (PLK-1) inhibitor BI2536 (Sur et al, 2009). In addition, xenograft studies showed that pretreatment with nutlin-3 did not interfere with the anti-tumour effect of BI2536. This is a remarkable result and moves the potential use of nutlin-3 for the application of cyclotherapy closer to the clinic.

ACTINOMYCIN D, AN OLD CHEMOTHERAPY DRUG, FOR P53-BASED CYCLOTHERAPY

Actinomycin D (ActD), like nutlin-3, is a potent activator of p53. ActD was the first antibiotic shown to have anti-tumour activity. This old chemotherapy agent had been used in the clinic for the treatment of children with rhabdomyosarcoma, Wilms' tumour and Hodgkin's disease (Farber et al, 1960). Low, nanomolar, doses of ActD are not highly genotoxic (Foster et al, 1994), effectively increase p53 levels and transcription function and induce the expression of a panel of genes that overlaps with nutlin3 -induced genes (Choong et al, 2009). In addition, this highly potent compound can cause p53-dependent reversible cell cycle arrest in normal keratinocytes (Choong et al, 2009).

Despite the apparent similarities between the effects of ActD and nutlin-3 in cells, the mechanisms of action are very different. Whereas nutlin-3 directly binds to $\mathrm{Mdm} 2$, ActD inhibits RNA synthesis by binding to GC-rich regions in DNA and is especially effective at disrupting ribosomal RNA biosynthesis (Goldberg, 1971). This causes a nucleolar stress that is sensed by p53 (Rubbi and Milner, 2003). According to the current model, nucleolar stress caused by $\mathrm{ActD}$ is able to enhance the interaction between Mdm2 and ribosomal proteins, such as L11, resulting in the impairment of Mdm2-dependent degradation of p53 (Sundqvist et al, 2009).

Encouraged by the remarkable results obtained by combining nutlin-3 with S- and M-phase poisons and the similar p53-dependent effects of low-dose ActD and nutlin-3, we have investigated ActD for application as cyclotherapy. We found that low, non-genotoxic, doses of ActD can induce a reversible cytostatic effect in normal proliferating fibroblasts and protect them from the aneuploidy induced by VX-680 (Rao et al, 2010). However, pretreating cells bearing mutant $p 53$ or lacking $p 53$ with this low dose of ActD also prevented the appearance of polyploidy in these cells. Even so, this pretreatment did not weaken the effects of VX-680 in clonogenic assays (Rao et al, 2010).

Subsequently, we investigated ActD in combination with the nucleoside inhibitor gemcitabine. Here, we found that pretreating with low, non-genotoxic doses of ActD, prior to adding gemcitabine, does not protect tumour cells bearing mutant p53 or lacking $p 53$. Pretreating normal proliferating fibroblasts, or wild-type p53 tumour cells with low doses of ActD, indeed protected these from the toxic effects of gemcitabine (van Leeuwen et al, 2012).

Thus, ActD as a clinically approved drug is an exciting candidate for cyclotherapy. However, further studies using in vivo models need to be developed before this approach can reach clinical practice.

P53-BASED CYCLOTHERAPY FOR BREAST CANCER: A CLINICAL PERSPECTIVE

Breast cancer survival is increasing due to early detection and advances in treatment. However, there remains a need for more effective and better tolerated treatments. Chemotherapy is used as 
adjuvant treatment for early-stage invasive breast cancer to prevent recurrence and is also used to treat advanced breast cancer.

Triple negative breast cancers (TNBCs) are defined as tumours that lack expression of oestrogen receptor (ER), progesterone receptor (PR) and HER2 receptor. Triple negative breast cancers are aggressive and therapeutically challenging breast cancers currently treated with a combination therapy involving surgery, radiotherapy and chemotherapy. Triple negative breast cancer account for $17 \%$ of all breast cancers. Prominent features of TNBC include overexpression of epidermal growth factor receptor (EGFR), a high proliferative rate, frequent genomic alterations, phenotypic similarity to BRCA1-associated cancers and frequent mutations of the $p 53$ gene with the corresponding protein heterogeneously expressed. The established chemotherapy for triple negative breast cancer includes combination chemotherapy including anthracyclines such as doxorubicin or epirubicin, taxanes such as paclitaxel or docetaxel and platinum drugs such as carboplatin and cisplatin (Torrisi et al, 2008).

Studies have suggested that patients with TNBC may benefit from high-dose or intensified chemotherapy (weekly or bi-weekly) regimens (Gluz et al, 2008). Dose-intensive chemotherapy may result in higher side effects compared to the less-intensive regimens. Side effects generally include alopaecia, neutropaenia and diarrhoea. A p53-based cyclotherapy approach could be applied in this setting to continue intensified treatments to tackle aggressive p53-mutant TNBC. While using p53 activators for the purposes of cyclotherapy, a major consideration has to be p53-related toxicities to normal tissues. Past studies using preclinical models have indicated specific toxicity only in tissues harbouring wild-type p53 during cytotoxic therapy (Komarov et al, 1999). In order to enhance the therapeutic window using cyclotherapy, specific and highly selective $p 53$ activators that can induce a mild 'cytostatic' effect will need to be carefully selected.

\section{FUTURE DIRECTIONS}

A remarkable in vivo study showing a reduction in neutropaenia in mice after pretreatment with nutlin-3 provides great optimism for p53-based cyclotherapy (Sur et al, 2009). However, Nutlin-3 has yet to be clinically approved. A nutlin-like compound, RG7112, has been tested in patients with mdm2-amplified liposarcomas (RayCoquard et al, 2012). While one patient achieved partial remission and 14 stable disease among the 20 treated, this study also highlighted that all of the patients who received neoadjuvant therapy with RG7112 experienced severe side effects including neutropaenia. These side effects may have been observed perhaps due to the high doses $\left(1440 \mathrm{mg} \mathrm{m}^{-2} \mathrm{day}^{-1}\right)$ used. Based on these observations, it cannot be ruled out that there may be many limitations for nutlin use in the clinic for the purposes of cyclotherapy.

Recently, Brown et al (2013) have developed stapled peptides that are highly specific inhibitors of $\mathrm{Mdm} 2 / \mathrm{MdmX}$. These compounds are more potent in activating p53 than peptides derived from a wild-type $p 53$ sequence. Unlike nultin-3, these peptides can sustain p53 activity over a wider range of concentrations without much toxicity to the cells. This suggests that these peptides could be ideal candidates for future cyclotherapy.

ActD, unlike nutlin-3, is a clinically approved drug with promise for cyclotherapy along-side S-phase but not mitotic poisons (Rao et al, 2010; van Leeuwen et al, 2012). A recent investigation of 16 different cyclotherapy regimes using nutlin-3, ActD, leptomycin $\mathrm{B}$ and tenovin 6 as cyclotherapeutic drugs in combination with the S-phase poisons, gemcitabine and cytosine arabinoside and the M-phase poisons, vinorelbine and vinblastine, highlights the need to assess both short-term and long-term effects of these combinations (van Leeuwen et al, 2012).

Approval of cyclotherapy in the clinic requires the development of in vivo models. We are investigating the effects of ActD in vivo to deduce the interval of time during which growth arrest can be established in mice gut crypt cells. This interval may include a window of opportunity to add an S-phase-specific drug that can then specifically target only tumour cells sparing the cells of gut crypts, one target of conventional chemotherapy approaches. It is critical to test the cyclotherapy combinations on different normal tissues to understand tissue-specific pathological effects. The p53 activators currently being tested for cyclotherapy all have distinct mechanisms to activate p53 and need to be tested in different combinations in vivo. The future of cyclotherapy holds exciting promise for targeting cancer cells while sparing normal tissues in a way currently not achieved with conventional chemotherapy.

\section{REFERENCES}

Agarwal ML, Agarwal A, Taylor WR, Stark GR (1995) p53 controls both the G2/M and the G1 cell cycle checkpoints and mediates reversible growth arrest in human fibroblasts. Proc Natl Acad Sci USA 92(18): 8493-8497.

Blagosklonny MV (2002) Sequential activation and inactivation of G2 checkpoints for selective killing of p53-deficient cells by microtubuleactive drugs. Oncogene 21(41): 6249-6254.

Blagosklonny MV, Pardee AB (2001) Exploiting cancer cell cycling for selective protection of normal cells. Cancer Res 61(11): 4301-4305.

Blagosklonny MV, Robey R, Bates S, Fojo T (2000) Pretreatment with DNA-damaging agents permits selective killing of checkpoint-deficient cells by microtubule-active drugs. J Clin Invest 105(4): 533-539.

Brown CJ, Quah ST, Jong J, Goh AM, Chiam PC, Khoo KH, Choong ML, Lee MA, Yurlova L, Zolghadr K, Joseph TL, Verma CS, Lane DP (2013) Stapled peptides with improved potency and specificity that activate p53. ACS Chem Biol 8(3): 506-512.

Bunz F, Dutriaux A, Lengauer C, Waldman T, Zhou S, Brown JP, Sedivy JM, Kinzler KW, Vogelstein B (1998) Requirement for p53 and p21 to sustain G2 arrest after DNA damage. Science 282(5393): 1497-1501.

Carvajal D, Tovar C, Yang H, Vu BT, Heimbrook DC, Vassilev LT (2005) Activation of $\mathrm{p} 53$ by MDM2 antagonists can protect proliferating cells from mitotic inhibitors. Cancer Res 65(5): 1918-1924.

Choong ML, Yang H, Lee MA, Lane DP (2009) Specific activation of the p53 pathway by low dose actinomycin D: a new route to $\mathrm{p} 53$ based cyclotherapy. Cell Cycle 8(17): 2810-2818.

Donehower LA, Godley LA, Aldaz CM, Pyle R, Shi YP, Pinkel D, Gray J, Bradley A, Medina D, Varmus HE (1996) The role of p53 loss in genomic instability and tumor progression in a murine mammary cancer model. Prog Clin Biol Res 395: 1-11.

Dulic V, Stein GH, Far DF, Reed SI (1998) Nuclear accumulation of p21Cip1 at the onset of mitosis: a role at the G2/M-phase transition. Mol Cell Biol 18(1): 546-557.

Farber S, D’Angio G, Evans A, Mitus A (1960) Clinical studies on actinomycin D with special reference to Wilms' tumor in children. Ann N Y Acad Sci 89: 421-425.

Foster SA, Demers GW, Etscheid BG, Galloway DA (1994) The ability of human papillomavirus E6 proteins to target $\mathrm{p} 53$ for degradation in vivo correlates with their ability to abrogate actinomycin D-induced growth arrest. J Virol 68(9): 5698-5705.

Gluz O, Nitz UA, Harbeck N, Ting E, Kates R, Herr A, Lindemann W, Jackisch C, Berdel WE, Kirchner H, Metzner B, Werner F, Schutt G, Frick M, Poremba C, Diallo-Danebrock R, Mohrmann S (2008) Triple-negative high-risk breast cancer derives particular benefit from dose intensification of adjuvant chemotherapy: results of WSG AM-01 trial. Ann Oncol 19(5): 861-870.

Goldberg IH (1971) The interaction of actinomycin with DNA. Antibiot Chemother 17: 67-86.

Isobe M, Emanuel BS, Givol D, Oren M, Croce CM (1986) Localization of gene for human p53 tumour antigen to band 17p13. Nature 320(6057): $84-85$.

Komarov PG, Komarova EA, Kondratov RV, Christov-Tselkov K, Coon JS, Chernov MV, Gudkov AV (1999) A chemical inhibitor of p53 that 
protects mice from the side effects of cancer therapy. Science 285(5434): 1733-1737.

Kranz D, Dobbelstein M (2006) Nongenotoxic p53 activation protects cells against S-phase-specific chemotherapy. Cancer Res 66(21): 10274-10280.

Lane DP (1992) Cancer. p53, guardian of the genome. Nature 358(6381): $15-16$.

Malkin D, Li FP, Strong LC, Fraumeni Jr JF, Nelson CE, Kim DH, Kassel J, Gryka MA, Bischoff FZ, Tainsky MA, Friend SH (1990) Germ line p53 mutations in a familial syndrome of breast cancer, sarcomas, and other neoplasms. Science 250(4985): 1233-1238.

Niculescu 3rd AB, Chen X, Smeets M, Hengst L, Prives C, Reed SI (1998) Effects of p21(Cip1/Waf1) at both the G1/S and the G2/M cell cycle transitions: $\mathrm{pRb}$ is a critical determinant in blocking DNA replication and in preventing endoreduplication. Mol Cell Biol 18(1): 629-643.

Rao B, van Leeuwen IM, Higgins M, Campbell J, Thompson AM, Lane DP, Lain S (2010) Evaluation of an Actinomycin D/VX-680 aurora kinase inhibitor combination in p53-based cyclotherapy. Oncotarget 1(7): 639-650.

Ray-Coquard I, Blay JY, Italiano A, Le Cesne A, Penel N, Zhi J, Heil F, Rueger R, Graves B, Ding M, Geho D, Middleton SA, Vassilev LT, Nichols GL, Bui BN (2012) Effect of the MDM2 antagonist RG7112 on the P53 pathway in patients with MDM2-amplified, well-differentiated or dedifferentiated liposarcoma: an exploratory proof-of-mechanism study. Lancet Oncol 13(11): 1133-1140.

Rubbi CP, Milner J (2003) Disruption of the nucleolus mediates stabilization of p53 in response to DNA damage and other stresses. EMBO J 22(22): 6068-6077.

Sundqvist A, Liu G, Mirsaliotis A, Xirodimas DP (2009) Regulation of nucleolar signalling to 553 through NEDDylation of L11. EMBO Rep 10(10): 1132-1139.
Sur S, Pagliarini R, Bunz F, Rago C, Diaz Jr. LA, Kinzler KW, Vogelstein B, Papadopoulos N (2009) A panel of isogenic human cancer cells suggests a therapeutic approach for cancers with inactivated p53. Proc Natl Acad Sci USA 106(10): 3964-3969.

Toledo F, Wahl GM (2006) Regulating the p53 pathway: in vitro hypotheses, in vivo veritas. Nat Rev Cancer 6(12): 909-923.

Torrisi R, Balduzzi A, Ghisini R, Rocca A, Bottiglieri L, Giovanardi F, Veronesi P, Luini A, Orlando L, Viale G, Goldhirsch A, Colleoni M (2008) Tailored preoperative treatment of locally advanced triple negative (hormone receptor negative and HER2 negative) breast cancer with epirubicin, cisplatin, and infusional fluorouracil followed by weekly paclitaxel. Cancer Chemother Pharmacol 62(4): 667-672.

van Leeuwen IM, Rao B, Sachweh MC, Lain S (2012) An evaluation of small-molecule p53 activators as chemoprotectants ameliorating adverse effects of anticancer drugs in normal cells. Cell Cycle 11(9): 1851-1861.

Vassilev LT, Vu BT, Graves B, Carvajal D, Podlaski F, Filipovic Z, Kong N, Kammlott U, Lukacs C, Klein C, Fotouhi N, Liu EA (2004) In vivo activation of the p53 pathway by small-molecule antagonists of MDM2. Science 303(5659): 844-848.

Vogelstein B, Lane D, Levine AJ (2000) Surfing the p53 network. Nature 408(6810): 307-310.

Weber JD, Taylor LJ, Roussel MF, Sherr CJ, Bar-Sagi D (1999) Nucleolar Arf sequesters Mdm2 and activates p53. Nat Cell Biol 1(1): 20-26.

(c) (1) (2) (2) This work is licensed under the Creative Commons cy NG SA Attribution-NonCommercial-Share Alike 3.0 Unported License. To view a copy of this license, visit http://creativecommons. org/licenses/by-nc-sa/3.0/ 